\title{
Geographical Variation in Opioid Use in Elderly Patients with Dementia: A Nationwide Study
}

\author{
Christina Jensen-Dahm ${ }^{\mathrm{a}, *}$, Johanne Købstrup Zakarias ${ }^{\mathrm{a}}$, Christiane Gasse ${ }^{\mathrm{b}, \mathrm{c}}$ and Gunhild Waldemar ${ }^{\mathrm{a}}$ \\ ${ }^{a}$ Danish Dementia Research Centre, Department of Neurology, Rigshospitalet, \\ University of Copenhagen, Copenhagen, Denmark \\ ${ }^{\mathrm{b}}$ Depression and Anxiety/Psychosis Research Unit, Aarhus University Hospital Psychiatry, Aarhus N, Denmark \\ ${ }^{\mathrm{c}}$ National Centre for Register Based Research, Aarhus University, Aarhus, Denmark
}

Handling Associate Editor: Alba Malara

Accepted 8 June 2019

\begin{abstract}
.
Background: We recently reported frequent use of opioids among elderly with dementia. Discrepancies in clinical practice may in part explain the higher use of opioids in elderly with dementia, which geographical variation may be able to clarify. Objective: To investigate geographical variation in opioid use in elderly with dementia compared to elderly without dementia. Methods: Register-based cross-sectional study in the entire elderly ( $\geq 65$ years) population of Denmark in 2015. Data included place of residence, prescriptions, and discharge diagnoses from hospital contacts. Prevalence of opioid use among elderly with $(n=36,014)$ and without dementia $(n=1,011,787)$ was compared nationwide across the five Danish regions using logistic regression analysis and for the 98 municipalities using age and sex standardization.

Results: $32.5 \%$ of elderly with dementia and $16.9 \%$ without were treated with an opioid in 2015 . For home-living elderly with dementia, there was a 4-fold difference in opioid use (9.4 to 36.8\%) between municipalities compared to a 1.6-fold (12.7 to $20.2 \%$ ) difference for elderly without. In nursing home residents there was a 2-fold difference (dementia: 26.5 to $55.2 \%$; no dementia: 31.8 to $60.4 \%$ ). Differences between the five regions were minor.

Conclusion: Opioid use in elderly with dementia was frequent and almost twice as high compared to elderly without dementia, which may challenge patient safety. The pronounced geographical variations at municipality level, particularly among elderly with dementia, indicate differences in the approach to treatment of chronic pain in primary care. Our study suggests that more guidance on treatment of pain in elderly with dementia is needed.
\end{abstract}

Keywords: Analgesics, dementia, elderly, opioid, pain

\section{INTRODUCTION}

Multimorbidity is frequent in the elderly population and pain causing conditions are frequent [1]. Thus, appropriate use of drugs and management of

\footnotetext{
*Correspondence to: Christina Jensen-Dahm, MD, PhD, Danish Dementia Research Centre, Department of Neurology, Neuroscience Centre, Rigshospitalet, University of Copenhagen, Blegdamsvej 9, \#6911, 2100 Copenhagen $\emptyset$, Denmark. Tel.: +45 35456911; E-mail: christina.jensen-dahm@regionh.dk.
}

comorbidities represent an important public health issue. Patients with dementia are less likely to report pain [2-4], but a recent register-based study found that use of opioids were frequent among elderly with dementia [5]. The reasons for the frequent use of opioids in elderly with dementia were unknown and could not be explained by differences in prevalence of pain-causing conditions. Use of opioids may be particularly problematic in patients with dementia due to sedation as well as possible cognitive impairment [6]. 
Discrepancies in clinical practice and lack of adherence to guidelines may in part explain the frequent use of opioids in elderly with dementia. These discrepancies may be clarified by studying geographical variation. Recently, we demonstrated profound geographical variation in the use of antipsychotics in elderly with dementia with a 4-fold difference in the prevalence of antipsychotic users between municipalities, even between municipalities within the same region [7]. At present, it is unknown if regional variation exists regarding opioid use in elderly with and without dementia. Danish registries are unique with respect to capturing an entire population and featuring detailed information on drug utilization. Knowledge about geographical variation in the Danish population may provide further insight into the extent and nature of variation in clinical practice in the care of pain in elderly with dementia also relevant in an international context. Thus, to determine possible factors contributing to the frequent consumption of opioids among elderly with dementia in Denmark, we aimed to investigate potential geographical variation in opioid use among elderly with and without dementia.

\section{MATERIALS AND METHODS}

\section{Study design and ethics}

The study was designed as an observational crosssectional study in 2015 using data from nationwide Danish registries. The study was approved by the Danish Data Protection Agency (ID no: 2007-580015/30-0667), Statistics Denmark, and the Danish Health and Medicine Authority (ID no: 6-8011907/1). Danish law does not require ethics committee approval or written informed patient consent for register based studies.

\section{The registers}

In Denmark, all permanent residents are assigned a personal identification number (CPR number) at time of birth or immigration, which makes it possible to link demographic and medical data from registers at an individual level [8]. The National Patient Register contains data on all hospital admissions since 1977 and all contacts to outpatient clinics and emergency rooms since 1995 [9]. The Psychiatric Central Research Register contains data on all admissions to psychiatric hospitals since April 1, 1969 and all outpatient contacts since 1995 [10]. The diagnoses have been registered from 1970-1993 according to the World Health Organization's (WHO) International Classification of Diseases 8th Revision (ICD-8) [11] and 10th Revision (ICD-10) from 1994 onwards [12]. The Danish National Prescription Register contains data on all dispensed prescription drugs since 1995 . The drugs are registered according to the Anatomical Therapeutic Chemical (ATC) classification system, with information on the date of dispensing, package size, and strength [13].

\section{Demographic information}

Denmark is divided into five administrative regions and 98 municipalities. The regions are responsible for hospitals and for private practice specialists and general practitioners (GPs). The municipalities are local government bodies responsible for primary care including home care and nursing homes for the elderly. The place of residence (region and municipality) on January 1, 2015, was identified using the Danish Civil Registration System [8], while information on living status (nursing homes) was retrieved from Statistics Denmark. Information about residence was used to examine geographical variation. Due to very few inhabitants at four Danish municipality islands (Fan $\varnothing$, Ærø, Sams $\varnothing$, and Læs $\varnothing$ ), we choose to exclude these from the analysis of municipalities.

\section{Study population}

All Danish residents aged $\geq 65$ years and alive on January 1, 2015, were identified using the Danish Civil Registration System [8]. Information about age, sex, and marital status was retrieved from the Danish Civil Registration System. Patients with a dementia diagnosis were identified as those who had been registered with a dementia diagnosis in the National Patient Register or Psychiatric Central Research Register before January 1, 2015, during admission or at an outpatient visit (Supplementary Table 1 for diagnostic codes) and/or those who had filled an anti-dementia drug prescription (ATC: N06D). The individuals had to be $\geq 60$ years at the time of the dementia diagnosis and/or first prescription because the validity of the diagnosis in those $<60$ years has been shown to be low [14]. The validity of a registered dementia diagnosis in those $\geq 65$ years, in contrast, 
has previously been shown to be high [15]. The remaining individuals formed the reference group.

\section{Opioid use}

In Denmark, opioids are only available by prescription from a physician and can only be dispensed once per prescription. Opioid users were defined as individuals who had redeemed at least one opioid prescription (ATC: N02A) in 2015.

\section{Comorbidity}

Comorbidity was evaluated at baseline (January 1 , 2015). Data from somatic and psychiatric hospital contacts was used to identify comorbid conditions. We evaluated potentially pain-causing conditions (cancer, osteoporosis, arthritis, and recent fracture) and comorbidity (diabetes, vascular, pulmonary, renal, and liver disease) that may affect opioid use (Supplementary Table 2 for diagnostic codes). The total amount of comorbidity was evaluated using the Charlson Comorbidity Index, which comprises 19 chronic somatic diseases and was used to assess comorbidity [24]. The total number of different drugs used in 2014 was employed as a surrogate marker of somatic disease not captured by hospital admissions (ATC level 3, e.g., A10A: insulins and analogues). Statistics Denmark provided information about living status (home-living and nursing home).

\section{Statistical analysis}

Normality of continuous variables was graphically determined using histograms. Differences in baseline characteristics was evaluated using Pearson's chi-square test. Logistic regression analyses were performed to evaluate the association between opioid use and dementia and effect of region (crude analysis). Furthermore, we adjusted for age, sex, and comorbidity (individual conditions) (adjusted analysis) as these differ between the regions. The percentage of opioid users in the various municipalities was adjusted using direct standardization to account for differences in age and sex between geographical areas and numbers are given as standardized values. A $p$-value of $<0.05$ was considered statistically significant. The data analysis was performed using SAS statistical software, version 9.4 (SAS Institute Inc., Cary, NC, USA).

\section{RESULTS}

\section{Study population}

The selection of the study population is shown in Fig. 1. In 2015, the population consisted of $1,047,801$ persons aged 65 and older, among whom 36,014 (3.4\%) were diagnosed with dementia. The elderly with dementia were older, more likely to be female, received more medication, and were suffering from more comorbidity (see Table 1 for details of the population). When stratified by region, there were significant differences in frequency of comorbidity among both elderly with and without dementia (see Supplementary Table 3A, B).

\section{National prevalence of opioid use}

$32.5 \%$ of elderly with dementia and $16.9 \%$ without dementia were treated with an opioid in 2015. Among the home-living, $24.0 \%$ of elderly with dementia and $16.1 \%$ of elderly without dementia were treated with an opioid in 2015 (see Table 2). Prevalence of opioid use was higher in nursing home residents among whom $39.6 \%$ of elderly with dementia and $44.3 \%$ of elderly without dementia were treated with an opioid in 2015.

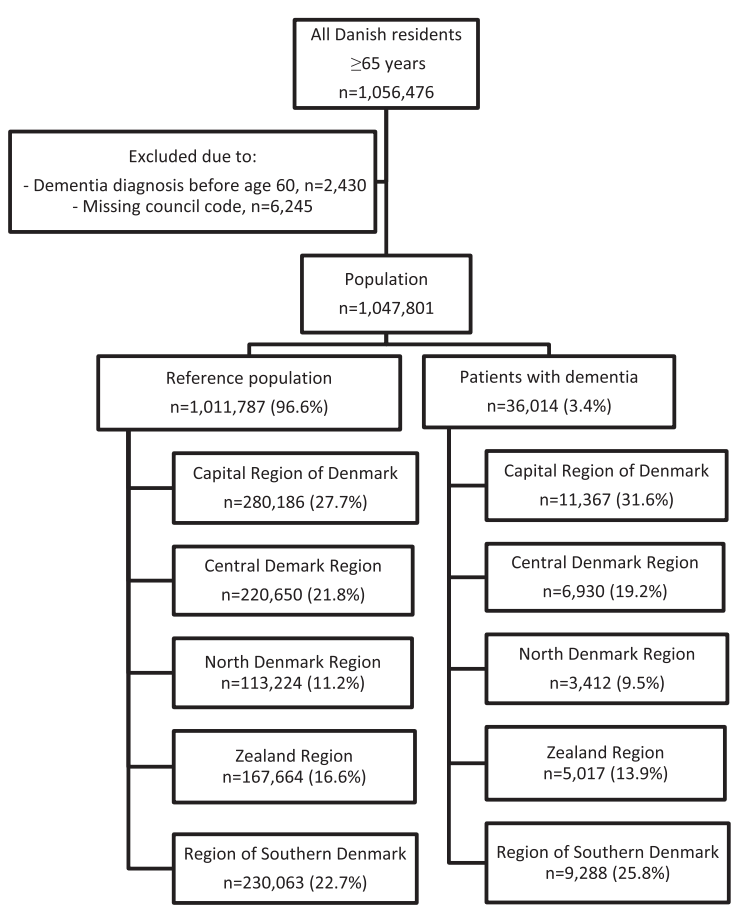

Fig. 1. Population selection. 
Table 1

Baseline characteristics stratified by dementia diagnosis

\begin{tabular}{lccc}
\hline Characteristics & $\begin{array}{c}\text { Dementia } \\
(\mathrm{N}=36,014)\end{array}$ & $\begin{array}{c}\text { Reference } \\
(\mathrm{N}=1,011,787)\end{array}$ & $p$ \\
\hline Age, y & $82.7(7.4)$ & $74.4(7.2)$ & \\
Female & $22,779(63.3 \%)$ & $548,160(54.2 \%)$ & $<0.0001$ \\
Married & $12,726(35.3 \%)$ & $56.6 \%$ & $<0.0001$ \\
Nursing home resident & $19,718(54.8 \%)$ & $30,650(3.0 \%)$ & $<0.0001$ \\
Time since dementia diagnosis & $3.0(1.3-5.4)$ & $\mathrm{NR}$ & \\
Charlson comorbidity index & $2.0(1.0-3.0)$ & $0.0(0.0-2.0)$ & $<0.0001$ \\
Total number of drugs used & $8.0(6.0-12.0)$ & $5.0(3.0-9.0)$ & $<0.0001$ \\
Antidepressant use in 2014 & $17,371(48.2 \%)$ & $117,229(11.6 \%)$ & $<0.0001$ \\
Pain causing comorbidity & & & \\
- Osteoporosis & $6,815(18.9 \%)$ & $116,283(11.5 \%)$ & $<0.0001$ \\
- Cancer & $7,888(21.9 \%)$ & $206,519(20.4 \%)$ & $<0.0001$ \\
- Diabetes & $4,466(12.4 \%)$ & $84,942(8.4 \%)$ & $<0.0001$ \\
- Fracture in 2014 & $2,885(8.0 \%)$ & $27,919(2.8 \%)$ & $<0.0001$ \\
- Arthritis & $7,130(19.8 \%)$ & $160,571(15.9 \%)$ & $<0.0001$ \\
Other comorbidity & & & \\
- Vascular disease & $14,453(40.1 \%)$ & $229,775(22.7 \%)$ & $<0.0001$ \\
- Pulmonary disease & $5,011(13.9 \%)$ & $108,710(10.7 \%)$ & $<0.0001$ \\
- Liver disease & $709(2.0 \%)$ & $13,828(1.4 \%)$ & $<0.0001$ \\
- Kidney disease & $1,970(5.5 \%)$ & $37,284(3.7 \%)$ & $<0.0001$ \\
\hline
\end{tabular}

Numbers are given as median (25-75\% interquartile range) for data with a non-normal distribution. Frequency numbers are given as percentage.

Table 2

Prevalence of opioid use (total) stratified by living status and dementia diagnosis

\begin{tabular}{lcclcc}
\hline & \multicolumn{2}{c}{$\begin{array}{c}\text { Home living } \\
(n=1,009,147)\end{array}$} & & \multicolumn{2}{c}{$\begin{array}{c}\text { Nursing home } \\
(n=38,654)\end{array}$} \\
\cline { 2 - 3 } & $\begin{array}{c}\text { Dementia } \\
(n=19,650)\end{array}$ & $\begin{array}{c}\text { Reference } \\
(n=989,497)\end{array}$ & & $\begin{array}{c}\text { Dementia } \\
(n=16,364)\end{array}$ & $\begin{array}{c}\text { Reference } \\
(n=22,290)\end{array}$ \\
\hline Capital Region of Denmark & $1,188(23.7 \%)$ & $42,952(15.8 \%)$ & & $2,559(40.3 \%)$ & $3,878(45.2 \%)$ \\
Central Denmark Region & $625(21.7 \%)$ & $34,561(16.2 \%)$ & & $1,476(36.4 \%)$ & $3,203(42.2 \%)$ \\
Northern Denmark Region & $281(21.3 \%)$ & $16,365(15.1 \%)$ & & $780(37.3 \%)$ & $1,892(42.4 \%)$ \\
Zealand Region & $682(27.2 \%)$ & $27,347(16.7 \%)$ & & $1,084(42.2 \%)$ & $1,815(46.1 \%)$ \\
Southern Region of Denmark & $1,139(24.9 \%)$ & $36,310(16.2 \%)$ & $1,906(40.4 \%)$ & $2,774(45.6 \%)$ \\
Total & $3,915(24.0 \%)$ & $157,535(16.1 \%)$ & $7,805(39.6 \%)$ & $13,562(44.3 \%)$ \\
\hline
\end{tabular}

\section{Regional prevalence of opioid use}

Table 2 shows the percentage of opioid users stratified by region, dementia diagnosis and living status. Opioid use ranged from 21.3\% (North Denmark Region) to $27.2 \%$ (Zeeland Region) $(p<0.0001)$ among home-living elderly with dementia and from $15.1 \%$ (North Denmark Region) to $16.7 \%$ (Zealand Region) $(p<0.0001)$ among home living elderly without dementia. For nursing home residents, opioid use ranged from $36.4 \%$ (Central Denmark Region) to $42.2 \%$ (Zeeland Region) $(p<0.0001)$ among elderly with dementia and from $42.2 \%$ (Central Denmark Region) to $45.6 \%$ (Region of Southern Denmark) $(p<0.0001)$ among elderly without dementia.

In a logistic regression of total opioid use, we adjusted for region to explore its effect on the estimate for dementia. Adjusting for region did not have an impact on the difference in opioid use in dementia versus no dementia in both the home-living and institutionalized elderly (see Supplementary Table 4A and $\mathrm{B}$ for details). Among home-living people, there were minor regional differences of opioid use (Central region: odds ratio ( $95 \%$ confidence interval): 1.08 (1.06-1.10); Zealand: $1.18(1.15-1.20))$ independent of dementia or comorbidity. Among the home-living adjusting for age and gender decreased the estimate for dementia from $1.65(1.59-1.72)$ to $1.28(1.23-1.33)$ and further to $1.12(1.08-1.17)$ after adjusting for comorbidity. For institutionalized people the estimate for dementia did not change significantly after adjusting for age, gender, and comorbidity.

\section{Prevalence of opioid use by municipality}

When opioid use was stratified by living status, it ranged from $9.4 \%$ (Favrskov municipality) to 

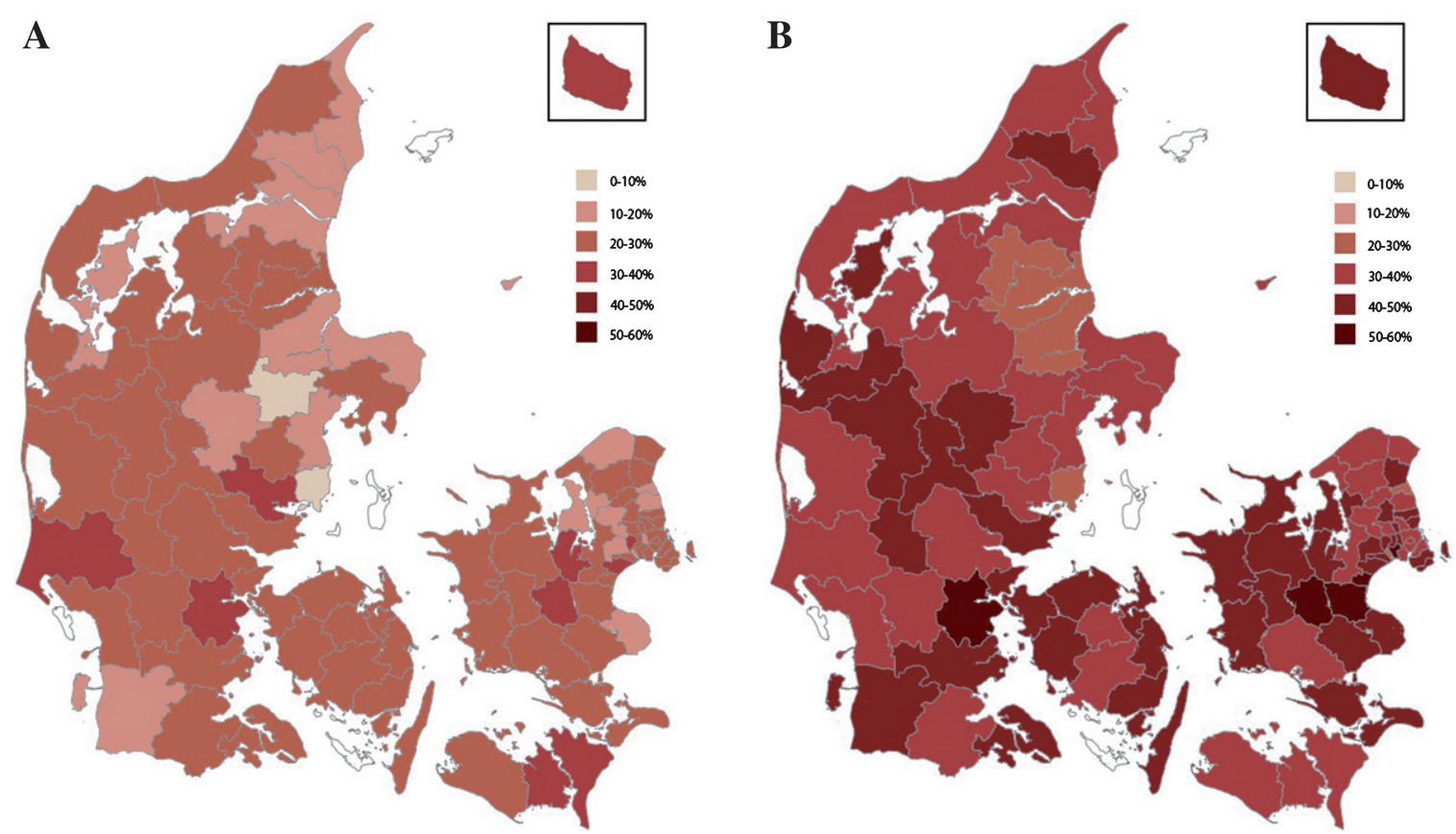

Fig. 2. A) Age and sex standardized prevalence of opioid use among home-living patients with dementia across the Danish municipalities. White indicates exclusion due to insufficient data. B) Age and sex standardized prevalence of opioid use among nursing home residents with dementia across the Danish municipalities. White indicates exclusion due to insufficient data.

$36.8 \%$ (Ringsted municipality) among elderly with dementia living at home (see Fig. 2A), yielding a 4-fold difference. For home-living elderly without dementia opioid use ranged from $12.7 \%$ (Allerød municipality) to $20.2 \%$ (Ishøj municipality), yielding a 1.6-fold difference. Among nursing home residents with dementia, opioid use ranged from $26.5 \%$ (Randers municipality) to $55.2 \%$ (Ringsted municipality) yielding a 2.1-fold difference (see Fig. 2B). For nursing home residents not diagnosed with dementia, opioid use ranged from $31.8 \%$ (Vallensbæk municipality) to $60.4 \%$ (Stevns municipality), yielding a 1.9-fold difference.

\section{DISCUSSION}

In this study of the entire elderly population of Denmark, the main finding was pronounced geographical variation in opioid use across municipalities. Among home-living elderly with dementia there was a 4-fold difference in opioid use, whereas there was a 2-fold difference in opioid use among nursing home residents with and without dementia.

To our knowledge, there have been no previous studies examining the geographical variation in opioid use among elderly with dementia. Previously, we demonstrated pronounced differences across municipalities in Denmark with a 4-fold difference in use of antipsychotics in elderly with dementia [7]. Geographical differences in use of antipsychotics have also been demonstrated in patients with schizophrenia and in the general population in the United States $[16,17]$. Large geographical variation in opioid use have been reported among the general population of Ontario with a 6-7 fold difference in opioid use between counties within Ontario [18]. In the US, a 2.7-fold difference between all states in opioid prescribing among the general population has been demonstrated [19]. None of the population studies examining use of opioids focused on elderly with dementia.

Previous population-based studies of opioid use in elderly with dementia have shown varied results. A study from Finland, which similar to ours was based on register-based data, found that in $20053.6 \%$ of community-dwelling elderly with Alzheimer's disease had used an opioid and $4.6 \%$ of communitydwelling elderly without dementia [20]. Using data from 2005 to 2011, another study from Finland investigated analgesic use in the first 180 days following diagnosis of Alzheimer's disease and found that opioids was used by $7.1 \%$ of elderly with Alzheimer's disease and by $8.1 \%$ of age- and sex 
matched controls [21]. However, the two studies from Finland investigated a very selected group as they examined home-living elderly, who had received an anti-dementia drug, thereby only including a subset of elderly with dementia. A recent Norwegian study used data from a cross-sectional study conducted in 2011 with 1,858 nursing home residents, where they extracted data from medication records and found that $23.8 \%$ were receiving an opioid [22]. Even though the studies did not use the exact same methodology, they demonstrated that there is significant variation in opioid use between countries with more than 3fold differences. Given the large variation between countries, we believe, that although our study was conducted in the elderly population of Denmark only, the extent and nature of the observed differences in the use of opioid is also relevant in an international context. We may only speculate about the reasons for the frequent use and pronounced geographical variation in elderly with dementia. Several factors may play a role, including differences in access to specialist care, local differences in management strategies and care of patients with pain, lack of knowledge about or uncertainty concerning assessment of pain in elderly with dementia as well as discrepancies to guidelines. Last, differences in degree of comorbidity may play a role.

Variation in opioid use was most pronounced at the municipal level, with a 4-fold difference for elderly with dementia living at home, by far exceeding the more modest variation between the larger regions. Hospitals are managed at a regional level, whereas primary care including home care and nursing homes are managed at the municipal level. As the variation in opioid use was most pronounced at the municipal level, this indicates that the explanation is related to discrepancies in clinical practice in primary care rather than differences in access to specialist care.

Several factors may explain the large variation among municipalities. There may be local differences in assessment and treatment of pain. Pain assessment in elderly with dementia is challenging, as the gold standard for pain assessment is 'the patient's report'. This becomes increasingly difficult as the disease progresses, and the patients lose the ability to communicate adequately [23, 24]. Therefore, among both the home-living and nursing home residents pain assessment and treatment in elderly with dementia depend very much on the caregiver's knowledge about the patient and interpretation of symptoms and the doctor may not have seen the patient when they made a prescription for an opioid. Thus, diffi- culty in assessment of pain and local differences in how potential 'pain indicators' are interpreted may be part of the explanation for why we found greater geographical variation among elderly with dementia compared to elderly without dementia. Furthermore, differences in knowledge and attitudes towards opioid use in elderly with dementia may also contribute to the geographical variation. A recent study found, that opioid prescribing in the emergency department was very dependent on the individual physician and opioid prescribing (in any age group and indication) varied by a factor 3.3, depending on the physician, even within the same hospital [25]. It is plausible that a similar inter-physician variation exists for the use of opioids for chronic pain in dementia. Thus, a lack of guidelines or lack of adherence to guidelines may also be part of the explanation. Alternatively, differences in degree of comorbidity may explain the variability. Adjusting for comorbidity decreased the overall estimate for the association between opioid prescription and dementia among elderly living at home; however, adjusting for region did not change the estimate for dementia neither in the crude nor the adjusted analysis. Age and sex had the greatest effect on the estimate for dementia. Within the municipalities, the prevalence's were standardized according to age and sex, but despite this, there was still a 2 to 4 -fold difference. We cannot exclude that differences in comorbidity explains part of the variability between municipalities, but it seems unlikely that this is the only explanation. The pronounced regional variation in prescription of opioids shows that the approach to treatment of pain in elderly with dementia is quite variable. One important question is which group is receiving the most optimal treatment, those with the highest or lowest opioid use? Previously, elderly with dementia were thought not to receive adequate treatment for pain as they received less analgesic medication compared to cognitively intact elderly [26, 27], but this does not seem to be the case in our sample. Recently, there has been increasing focus on the lack of evidence for effect of opioids in long-term treatment of chronic non-malignant pain $[28,29]$ and the significant risk associated with opioids [30,31]. Thus, recent guidelines recommend non-opioid therapy to treat chronic non-malignant pain [29, 32]. Guidelines for management in pain in the elderly states that opioids can be considered in carefully selected and monitored patients with moderate or severe pain, especially if causing functional impairment [33]. Dementia is not associated with an increased prevalence of pain but 
is associated with an increased risk of adverse events [34]. In a recent study of tolerability of buprenorphine in nursing home residents with advanced dementia, $52.3 \%$ discontinued treatment due to adverse events, compared to $13.3 \%$ in the placebo group. Buprenorphine reduced daytime activity and nursing home staff reported increased sedation/somnolence as the most frequent adverse events [35]. Thus, in treating pain in elderly with dementia opioids must be used according to safety guidelines especially in frail people such as elderly and dementia patients, considering the frequent occurrence of side-effect and potential for drug interactions in polytherapy.

\section{Limitations and strength}

The main strength of this study is its nationwide population-based design and high reliability of data. Our study investigated "real-life prescription patterns" in an entire elderly population, thus avoiding problems of selection bias. Previous research has shown that the validity of a dementia diagnosis in Danish hospital registries is high in the elderly population [15]. However, dementia is generally underdiagnosed and potentially undiagnosed cases of dementia could have been included in the reference population particularly in the nursing home population. Another limitation is that we do not have information about the severity of dementia or whether the patient had a current depression. We included information about time since diagnosis and found that median time since diagnosis for the home-living was 2.2 years and 3.6 years for the nursing home residents. Data on prescriptions for opioids filled at pharmacies were complete, but we did not have information about indications and whether patients consumed their prescriptions. As we wished to investigate differences between the 98 municipalities we were unable to look at subtypes of opioids or duration of opioid use due to very small groups in some municipalities but investigated this in a previous publication [5]. We included information about pain-causing conditions, but we do not have information about the type or degree of pain.

\section{Conclusion}

In the entire elderly population of Denmark, we found pronounced geographical variation in opioid use, which was not explained by differences in age, sex, and comorbidity. The differences were most pronounced among elderly with dementia at the municipality level, suggesting that the differences were primarily related to different approaches toward either pain assessment and/or pain treatment in primary care, home care as well as nursing homes. As opioid use differs between several nations, there are reasons to believe that our findings of within country variations may apply to other countries as well. This calls for more guidance on assessment and management of pain in elderly with dementia. Implementation of guidelines for appropriate management of pain in elderly with dementia is a challenging task that must be addressed jointly by targeting multiple audiences and not only the prescribing doctors. Future studies should explore potential consequences for patient safety.

\section{ACKNOWLEDGMENTS}

The Danish Dementia Research Centre is supported by grants from the Danish Ministry of Health (file no. 2007-12143-112/59506 and file no. 0901110/ 34501).

Authors' disclosures available online (https:// www.j-alz.com/manuscript-disclosures/19-0413r1).

\section{SUPPLEMENTARY MATERIAL}

The supplementary material is available in the electronic version of this article: http://dx.doi.org/ 10.3233/JAD-190413.

\section{REFERENCES}

[1] Sinnige J, Korevaar JC, Westert GP, Spreeuwenberg P, Schellevis FG, Braspenning JC (2015) Multimorbidity patterns in a primary care population aged 55 years and over. Fam Pract 32, 505-513.

[2] Achterberg WP, Gambassi G, Finne-Soveri H, Liperoti R, Noro A, Frijters DH, Cherubini A, Dell'aquila G, Ribbe MW (2010) Pain in European long-term care facilities: Cross-national study in Finland, Italy and The Netherlands. Pain 148, 70-74.

[3] Jensen-Dahm C, Vogel A, Waldorff FB, Waldemar G (2012) Discrepancy between self- and proxy-rated pain in Alzheimer's disease: Results from the Danish Alzheimer Intervention Study. J Am Geriatr Soc 60, 1274-1278.

[4] Scherder E, Bouma A, Borkent M, Rahman O (1999) Alzheimer patients report less pain intensity and pain affect than non-demented elderly. Psychiatry 62, 265-272.

[5] Jensen-Dahm C, Gasse C, Astrup A, Mortensen PB, Waldemar G (2015) Frequent use of opioids in patients with dementia and nursing home residents: A study of the entire elderly population of Denmark. Alzheimers Dement 11, 691699.

[6] Papaleontiou M, Henderson CR, Jr., Turner BJ, Moore AA, Olkhovskaya Y, Amanfo L, Reid MC (2010) Outcomes associated with opioid use in the treatment of chronic 
noncancer pain in older adults: A systematic review and meta-analysis. J Am Geriatr Soc 58, 1353-1369.

[7] Zakarias JK, Jensen-Dahm C, Norgaard A, Stevnsborg L, Gasse C, Andersen BG, Soren J, Waldorff FB, Moos T, Waldemar G (2016) Geographical variation in antipsychotic drug use in elderly patients with dementia: A nationwide study. J Alzheimers Dis 54, 1183-1192.

[8] Thygesen LC, Daasnes C, Thaulow I, Bronnum-Hansen H (2011) Introduction to Danish (nationwide) registers on health and social issues: Structure, access, legislation, and archiving. Scand J Public Health 39, 12-16.

[9] Lynge E, Sandegaard JL, Rebolj M (2011) The danish national patient register. Scand J Public Health 39, 30-33.

[10] Munk-Jorgensen P, Mortensen PB (1997) The Danish Psychiatric Central Register. Dan Med Bull 44, 82-84.

[11] Danish National Board of Health (1971) Classification of Diseases: The Danish Edition of ICD-8. Copenhagen.

[12] World Health Organization (1993) The ICD-10 classifcation of mental and behavioural disorders: Diagnostic criteria for research.

[13] Kildemoes HW, Sorensen HT, Hallas J (2011) The Danish National Prescription Registry. Scand J Public Health 39, 38-41.

[14] Salem LC, Andersen BB, Nielsen TR, Stokholm J, Jorgensen MB, Rasmussen MH, Waldemar G (2012) Overdiagnosis of dementia in young patients - a nationwide register-based study. Dement Geriatr Cogn Disord 34, 292299.

[15] Phung TK, Andersen BB, Hogh P, Kessing LV, Mortensen PB, Waldemar G (2007) Validity of dementia diagnoses in the Danish hospital registers. Dement Geriatr Cogn Disord 24, 220-228.

[16] King M, Essick C (2013) The geography of antidepressant, antipsychotic, and stimulant utilization in the United States. Health Place 20, 32-38.

[17] Stroup TS, Gerhard T, Crystal S, Huang C, Olfson M (2014) Geographic and clinical variation in clozapine use in the United States. Psychiatr Serv 65, 186-192.

[18] Gomes T, Juurlink D, Moineddin R, Gozdyra P, Dhalla I, Paterson M, Mamdani M (2011) Geographical variation in opioid prescribing and opioid-related mortality in Ontario. Healthc $Q$ 14, 22-24.

[19] Paulozzi LJ, Mack KA, Hockenberry JM (2014) Variation among states in prescribing of opioid pain relievers and benzodiazepines-United States, 2012. J Safety Res 51, 125-129.

[20] Bell JS, Laitinen ML, Lavikainen P, Lonnroos E, Uosukainen H, Hartikainen S (2011) Use of strong opioids among community-dwelling persons with and without Alzheimer's disease in Finland. Pain 152, 543-547.

[21] Hamina A, Taipale H, Tanskanen A, Tolppanen AM, Tiihonen J, Hartikainen S (2017) Differences in analgesic use in community-dwelling persons with and without Alzheimer's disease. Eur J Pain 21, 658-667.

[22] Sandvik R, Selbaek G, Kirkevold O, Aarsland D, Husebo BS (2016) Analgesic prescribing patterns in Norwegian nursing homes from 2000 to 2011: Trend analyses of four data samples. Age Ageing 45, 54-60.

[23] Hadjistavropoulos T, Herr K, Prkachin KM, Craig KD, Gibson SJ, Lukas A, Smith JH (2014) Pain assessment in elderly adults with dementia. Lancet Neurol 13, 1216-1227.

[24] Pautex S, Herrmann F, Le Lous P, Fabjan M, Michel JP, Gold G (2005) Feasibility and reliability of four pain selfassessment scales and correlation with an observational rating scale in hospitalized elderly demented patients. $J$ Gerontol A Biol Sci Med Sci 60, 524-529.

[25] Barnett ML, Olenski AR, Jena AB (2017) Opioidprescribing patterns of emergency physicians and risk of long-term use. $N$ Engl J Med 376, 663-673.

[26] Achterberg WP, Pieper MJ, van Dalen-Kok AH, de Waal MW, Husebo BS, Lautenbacher S, Kunz M, Scherder EJ, Corbett A (2013) Pain management in patients with dementia. Clin Interv Aging 8, 1471-1482.

[27] Scherder E, Herr K, Pickering G, Gibson S, Benedetti F, Lautenbacher S (2009) Pain in dementia. Pain 145, 276-278.

[28] Ballantyne JC (2017) Opioids for the treatment of chronic pain: Mistakes made, lessons learned, and future directions. Anesth Analg 125, 1769-1778.

[29] Dowell D, Haegerich TM, Chou R (2016) CDC guideline for prescribing opioids for chronic pain-United States, 2016. JAMA 315, 1624-1645.

[30] Hoffman EM, Watson JC, St Sauver J, Staff NP, Klein CJ (2017) Association of long-term opioid therapy with functional status, adverse outcomes, and mortality among patients with polyneuropathy. JAMA Neurol 74, 773-779.

[31] Ray WA, Chung CP, Murray KT, Hall K, Stein CM (2016) Prescription of long-acting opioids and mortality in patients with chronic noncancer pain. JAMA 315, 2415-2423.

[32] Danish National Board of Health (2018) Opioid behandling af kronisk ikke-maligne smerter [danish]; Opioid treatment for chronic non-malignant pain.

[33] Abdulla A, Adams N, Bone M, Elliott AM, Gaffin J, Jones D, Knaggs R, Martin D, Sampson L, Schofield P, British Geriatric S (2013) Guidance on the management of pain in older people. Age Ageing 42(Suppl 1), i1-i57.

[34] Barber JB, Gibson SJ (2009) Treatment of chronic nonmalignant pain in the elderly: Safety considerations. Drug Saf 32, 457-474.

[35] Erdal A, Flo E, Aarsland D, Selbaek G, Ballard C, Slettebo DD, Husebo BS (2018) Tolerability of buprenorphine transdermal system in nursing home patients with advanced dementia: A randomized, placebo-controlled trial (DEP.PAIN.DEM). Clin Interv Aging 13, 935-946. 